\title{
HISTORIA RECIENTE DE LA UNIVERSIDAD DE ALCALÁ (CONTINUACIÓN)
}

\section{RECENT HISTORY OF THE UNIVERSITY OF ALCALA (CONTINUED)}

\author{
AUTORES \\ José Morilla Critz: Catedrático de Universidad. Área de Fundamentos de la \\ Economía \\ jose.morilla@aol.com
}

\section{CURRÍCULUM VITAE}

Profesor en la Facultad de Ciencias Económicas y Empresariales. Universidad de Alcalá.

\section{RESUMEN}

Estos dos años asistieron a otra historia, tan rocambolesca como la anteriormente descrita, relacionada con la recuperación universitaria de Alcalá de Henares. De entrada, hemos de decir que los elementos que habían determinado la búsqueda de nuevos emplazamientos universitarios en Madrid hacía cuatro años persistían ahora e incluso se habían agravado, a pesar de la entrada en funcionamiento en 1971 de las nuevas instalaciones de la Universidad Autónoma. La masificación estudiantil se había agudizado y en la capital de España había ya 60.000 estudiantes universitarios (la mitad de los de España, aunque Madrid sólo tuviera aproximadamente el 10 por 100 de la población nacional). Por otra parte, las autoridades de la recién denominada "Complutense de Madrid", tal vez por este hecho, incrementaban su tendencia a buscar en Alcalá el "sello histórico" que les faltaba y, en cualquier caso, la 
idea de Alcalá había recorrido ciertos círculos de opinión gracias al gran debate de los años precedentes.

\title{
PALABRAS CLAVE
}

Historia - Universidad de Alcalá - Renacimiento

\begin{abstract}
These two years attended another story, as bizarre as described above, related to the recovery of Alcala de Henares University. To begin, we say that the elements which determined the search for new sites in Madrid university persisted for four years now and even had worsened, despite the entry into operation in 1971 of the new facilities at the Autonomous University. The student overcrowding had worsened and was the capital of Spain and 60,000 college students (half of those in Spain, but Madrid only had about 10100 of the national population). Moreover, the authorities of the newly named "Complutense de Madrid", perhaps because of this, increasing their tendency to look at Alcala the "seal historic" they lacked, and in any case, the idea of Alcala had traveled some circles of opinion due to the great debate of the previous years.
\end{abstract}

\section{KEY WORDS}

History - University of Alcalá - Renaissance

En esta ocasión, José Morilla nos ofrece la historia de las vicisitudes de la "casi" reapertura de la Universidad de Alcalá durante el trienio 1972-1974. Tal como se 
menciona en el último párrafo de este capítulo, es de agradecer a la corporación municipal de entonces que, por las razones que fueran, decidiera no ubicar un Instituto de Enseñanza Media en las instalaciones de la Base Aérea, dejando expedito el camino para la verdadera resurrección.

El Renacimiento de la Universidad de Alcalá (Por José Morilla Critz. Catedrático de Universidad. Área de Fundamentos de la Economía)

\section{1972-1974: nueva apertura y cierre de una universidad en Alcalá}

Estos dos años asistieron a otra historia, tan rocambolesca como la anteriormente descrita, relacionada con la recuperación universitaria de Alcalá de Henares. De entrada, hemos de decir que los elementos que habían determinado la búsqueda de nuevos emplazamientos universitarios en Madrid hacía cuatro años persistían ahora e incluso se habían agravado, a pesar de la entrada en funcionamiento en 1971 de las nuevas instalaciones de la Universidad Autónoma. La masificación estudiantil se había agudizado y en la capital de España había ya 60.000 estudiantes universitarios (la mitad de los de España, aunque Madrid sólo tuviera aproximadamente el 10 por 100 de la población nacional). Por otra parte, las autoridades de la recién denominada "Complutense de Madrid", tal vez por este hecho, incrementaban su tendencia a buscar en Alcalá el "sello histórico" que les faltaba y, en cualquier caso, la idea de Alcalá había recorrido ciertos círculos de opinión gracias al gran debate de los años precedentes.

La apertura del curso 1971-1972 en la Universidad de Madrid se realizó en el Paraninfo de Alcalá y le sirvió a Botella Llusiá, precisamente, para entonar un canto contra la insoportable masificación que soportaba esa universidad. Ya era común la preocupación, tanto de las autoridades universitarias como del Ministerio de Educación, de buscar nuevos "contenedores" para centros universitarios en Madrid. 
El Proyecto de III Plan de Desarrollo recogía en esos mismos años esta necesidad y previó para los siguientes cuatro la creación de seis nuevas universidades, entre las que se encontraba una más en Madrid. Por otra parte, en 1972 se había autorizado legalmente a las universidades, previa aceptación por el Ministerio de Educación y Ciencia, el "desdoblamiento" de facultades, es decir, que una misma universidad tuviera la posibilidad de abarcar en su seno más de un centro de la misma especialidad.

En Alcalá, miembros del grupo 69, como García Saldaña, aprovecharon la ocasión y se dirigieron al Jefe del Estado recordando la reivindicación alcalaína. Algunos medios de prensa, incluso de otras provinciasNota19, volvieron a hablar de Alcalá como conveniente sede universitaria. El Ayuntamiento complutense, contando también con el apoyo de numerosas industrias de la zona, inició una serie de escritos y visitas a los Ministerios, solicitando la "nueva universidad" para Alcalá. Ahora bien, tal era el desconocimiento que la Administración tenía de lo que había tras las reivindicaciones alcalaínas que, confundiendo su solicitud con el proyecto que el propio ministro de Educación tenía de integrar todas las escuelas técnicas de Madrid en un nuevo rectorado con el nombre de "Universidad Politécnica", contestó a aquellos requerimientos con una carta en la que decía:

no hay inconveniente en reponer esos centros en Alcalá (j?).

En este clima, en marzo de 1972, el nuevo equipo de gobierno de la Universidad Complutense, encabezado por el rector Ángel González Álvarez, pidió al Ayuntamiento de Alcalá que se le hiciera un informe sobre las posibilidades de situar en esta ciudad nuevos primeros y segundos cursos de facultades especialmente masificadas (en concreto, Físicas, Químicas, Biológicas y Filosofía y Letras). El Ayuntamiento no solamente hizo el informe, que se basaba en las posibilidades de los terrenos y edificaciones cedidos al Ministerio de Educación en su día, sino que 
mostró también su disposición a adquirir antiguas edificaciones. Y como botón de muestra, el Ayuntamiento cedería el antiguo convento de Dominicos (o también antigua "Cárcel del Partido") a la Universidad Complutense.

En visitas a Alcalá y reuniones con las autoridades locales, el Rectorado, con la promesa de apoyo económico por parte del Ministerio de Educación, llegó a la conclusión de que, a corto plazo - para el curso 1973-1974 -, la mejor opción era la utilización de las instalaciones de los Maristas en Alcalá de Henares para Facultad de Ciencias, una vez hechas las correspondientes obras de adaptación para aulas adecuadas y laboratorios. Previamente, se había comprobado la imposibilidad de instalarse en la Universidad Laboral y también se había constatado la necesidad de obras a más largo plazo en el antiguo campo de aviación que, no obstante, quedaba como proyecto para el futuro.

Las obras de adaptación del edificio de los Maristas se hicieron con notable celeridad durante el verano de 1973 y en septiembre, la Universidad Complutense y el Ministerio de Educación empezaron a enviar algún material y adscribieron o contrataron algún personal administrativo para la extensión de la Universidad Complutense, para cuyo desarrollo el director general de Universidades siempre pidió "mucha discreción"Nota20. Con todo ello, en septiembre de 1973 parecía que la apertura de centros de la "Complutense" en Alcalá era un hecho, puesto que el Rectorado insertó incluso los correspondientes anuncios de reserva de plazas:

\section{UNIVERSIDAD COMPLUTENSE. ALCALÁ DE HENARES. FACULTAD DE} CIENCIAS. CURSO 1973-1974: A partir de octubre, y como desdoblamiento de la Universidad Complutense, se impartirán las asignaturas correspondientes al primer año de la licenciatura de Ciencias en las nuevas Facultades de Física, Química, Biología. Reserva de plazas abierta en Alcalá de Henares, edificio de los $\mathrm{HH}$. 
Maristas. Las reservas de plaza efectuadas en Madrid pueden ser trasladadas a Alcalá a petición propia.

En Alcalá se podían ver en ese mismo mes de septiembre los rótulos indicadores de los centros universitarios y el Ayuntamiento convocaba becas para fomentar la investigación de los profesores que decidieran trasladarse a esos centros, además de un concurso periodístico sobre ideas de desarrollo de lo que para la ciudad era el embrión de retorno de la Complutense a su ciudad.

En este momento, por otra parte, el Rectorado de la Complutense era más consciente aún del problema de masificación que se le avecinaba: se encontró con una demanda de 24.000 nuevos alumnos para el próximo curso y se sabía impotente, ante la contestación estudiantil y la legalidad vigente, para rechazar ninguna solicitud. El rector, con el deseo de granjearse un apoyo estudiantil que en esos años no le era dado, en todo caso, a ninguna autoridad universitaria, declaraba que:

todo alumno que haya aprobado primero, segundo, tercero [...] de Bachillerato, hasta el sexto curso, y el COU (con la supervisión de la Universidad, no entro si buena o mala), no encontrará cerradas las puertas de la Universidad Complutense.

En esa tesitura planteó la necesidad de intensificar la política de desdoblar centros, programando hacerlo con diez de sus Facultades para el curso 1973-1974. En la solicitud al Ministerio se proponía que nueve de ellas se ubicaran en Alcalá de Henares, dada la disposición de terrenos que en esta ciudad tenía el Ministerio. La idea era que surgiría así una "Universidad Complutense" con dos "ciudades universitarias", una en Madrid y otra en Alcalá.

La respuesta del ministro Julio Rodríguez a los dos días fue la de una idea más "ambiciosa": crear la "cuarta Universidad de Madrid" y como para tal personaje la 
simplificación - o tal vez el miedo a la excesiva concentración de una masa contestataria - era un mal, anunció que la nueva universidad estaría repartida entre Madrid, Alcalá de Henares y Toledo, de tal manera que el Rectorado y la Facultad de Medicina estarían en Madrid, las carreras de Ciencias en Alcalá y las de Letras en Toledo. Como el curso no iba a empezar, según su nuevo calendario, hasta enero de 1974, habría tiempo para poner todo en marcha en el curso que empezaba.

Analizando las declaraciones de las partes implicadas en ambos proyectos (el de desdoblar Facultades en los Maristas, de la Complutense, y el de abrir centros de una nueva universidad, del Ministerio), resulta cuando menos interesante observar que hubo una auténtica pugna entre las mismas. El rector llegó a declarar, irritado, a una delegación del Ayuntamiento alcalaíno, que la decisión del Ministerio había sido hecha para colocar "fuera de combate" a la Universidad de Madrid, y el ministro Julio Rodríguez a otra delegación del mismo Ayuntamiento le preguntó: "¿Qué quieren, la cuarta universidad de Madrid o "seguir" con la Complutense?" Ante la pregunta de la delegación sobre qué implicaba cada opción, el ministro contestó: "La elección de la cuarta Universidad significa tener la Facultad de Ciencias, si eligen la Complutense no tendrán nada"Nota21. Esta "fiereza" entre un Rectorado y un Ministerio que, en definitiva, en aquellos tiempos dependía uno del otro, nos afirma en la sospecha de que el tema universitario de Alcalá era un instrumento más de lucha entre los "grupos de presión" del régimen en su última etapa.

Ante la nueva situación quedó paralizado el proyecto de desdoblamiento de la Complutense en Alcalá y el Ministerio de Educación concedió un pequeño crédito en octubre de 1973 para iniciar las obras de acondicionamiento de las edificaciones militares abandonadas en el campo de aviación, con la finalidad de que las clases de la "nueva universidad" comenzaran en enero de 1974. 
A finales de 1973, pues, nuevamente pareció a los alcalaínos que, de la forma que fuese, su tradición universitaria se iba a recuperar. Hasta tal punto estaba segura de ello la corporación municipal que, siguiendo el adulador rito de la dictadura, nombró "Alcalde Honorario Perpetuo" de Alcalá de Henares a Franco, en agradecimiento a que su régimen y Gobierno habían "devuelto" la Universidad a Alcalá. En el fondo ésta era la respuesta de los políticos locales leales al régimen a la militancia prouniversidad y contestataria que había mostrado en los años anteriores el grupo 69. Pero este homenaje y, sobre todo, el protocolario acto de nombramiento en el Pardo el 5 de diciembre, deberían ser recordados por las sucesivas generaciones de alcalaínos como uno de los más lamentables ridículos a los que unos políticos "leales" (como sólo puede haber en regímenes dictatoriales) llevaron el nombre de Alcalá, porque, llegado enero de 1974, la famosa "cuarta Universidad de Madrid" no se abrió y en abril de 1974, con un nuevo ministro, al asunto se le dio el carpetazo definitivo.

Esta fue, pues, una nueva esperanza frustrada de recuperación del papel universitario de Alcalá, pues nada se hizo y, en esta ocasión, ni tan siquiera hubo petición de disculpas o formulación de ambiguas promesas para el futuro. Aun cuando, vistas las cosas a posteriori, hay que reconocer que, como en el caso anterior, este abortado proyecto colaboró a facilitar las cosas para el inmediato futuro.

Como consecuencia del plan, paralizado a poco de comenzar, de esa cuarta y dispersa universidad de Madrid, se incrementó el valor "estratégico" de los terrenos de la base aérea de Alcalá ante los ojos de los futuros responsables del Ministerio de Educación, puesto que se habían realizado algunas inversiones en reformas que, desde ese momento, el propio Ministerio intentó utilizar para cualquier proyecto.

En segundo lugar, involucró a las autoridades locales (antes desconcertadas ante el protagonismo de los "contestatarios" del Grupo 69) en negociaciones efectivas ante 
diferentes organismos, de las que obtendrían un conocimiento muy útil para proceder en etapas sucesivas.

En tercer lugar, y esto sería muy interesante para lo que ocurriría más adelante, se plantearon ya de forma práctica programas de rehabilitación de edificios históricos para uso universitario de nuevoNota4 de VA.

Y, en cuarto lugar, Alcalá, como proyecto de recuperación universitaria, avanzó un poco más en cuanto a su imagen nacional, aunque fuera en círculos muy reducidos, que seguramente actuarían en su favor más adelante. Por primera vez también se planteó por algún ensayista la posibilidad de todo un programa recuperador:

Merecería la pena que la vuelta de la Universidad alcalaína se viera acompañada no sólo con el nombre de Complutense, que le pertenece, sino con un cuadro completo y el rescate de los viejos edificios existentes que rivalizaron en saberes durante el Siglo de Oro con la Universidad de Salamanca.

... bien importante sería apoyar desde este mismo momento la idea de conservar y restaurar todo el viejo sabor de aquella Complutense que tanto nombre dio a España más allá de sus fronteras. Junto a la emoción de los valores espirituales, junto a la esperanza de ese símbolo recobrado para la calle porticada y el aire de Alcalá, los valores estéticos, históricos, y literarios necesitan del mayor número de paredes, patios y puertas auténticos de nuestra cultura del Siglo de Oro.

La antigua sede de la Universidad Complutense, hoy dedicada a centro de formación y perfeccionamiento de funcionarios, debería recuperarse para rectorado y aula magna, lo mismo que otros edificios que pertenecen hoy al Ejército y que valdría la pena incorporar a esta Universidad recobrada. Aparte de las 230 hectáreas que están dispuestas, el viejo estilo universitario alcalaíno está en estas fachadas que acabo de 
visitar en un recorrido de urgencia, cuando Alcalá de Henares tiene la fiesta por dentro, para recibir "su" Universidad de hace ciento treinta y seis añosNota22.

Nuevamente conviene preguntarse por las razones de que no se cumplieran unas promesas de reinstalación universitaria en Alcalá. En este caso no hubo necesidad de declaraciones oficiales, aunque hubieran sido tan ambiguas como en el caso anterior, porque en realidad no había sido publicada ninguna resolución previa al respecto.

Dada la reiteración del Ministerio a partir de 1974 sobre las dificultades económicas, ante las peticiones de las autoridades locales, creemos que, reconocida ya la situación de crisis económica iniciada en 1973 (pero a la que el Gobierno había hecho oídos sordos al principio), se abandonaron las previsiones del III Plan de Desarrollo. Éste fue enterrado por el último gobierno de Franco, formado tras la muerte de Carrero Blanco, y presidido por Arias Navarro. Con ello se arrumbó la política de crear nuevas universidades como proyectos definidos y acabados, surgidas tras una planificación y dotadas de unos medios más o menos adecuados.

Por otra parte, a corto plazo, no hay que olvidar que la etapa de ministerio de Julio Rodríguez había sido tan excepcional en cuanto a las medidas universitarias adoptadasNota23, que sus decisiones adquirieron un gran descrédito, incluso en los medios más adeptos al régimen y en los círculos gubernamentales y de la administración.

Las primeras decisiones del sustituto de Julio Rodríguez, Cruz Martínez Esteruelas, fueron las de "restablecer" las condiciones de funcionamiento de la Universidad anteriores a la etapa "juliana" y, por tanto, todo proyecto en marcha hasta ese momento quedó completamente arrumbado. 
Por otra parte, como a la Universidad de Madrid no se le había autorizado en su día a abrir las instalaciones que había previsto en Alcalá de Henares y, sin embargo, no tenía capacidad en sus centros para alojar a los estudiantes, abrió Colegios Universitarios en la capital en condiciones muy precarias (en concreto, el Colegio "Arcos de Jalón", con instalaciones para Ciencias en el barrio de San Blas y para Económicas y Empresariales en la Casa de Campo) y ello parecía paliar los agobios que anteriormente sufrían.

En Alcalá de Henares, además, en estos años se empezó a sentir la necesidad de plazas de enseñanza primaria y media, como consecuencia del crecimiento de la población juvenil al cabo de diez años de intensa inmigración. Existía, pues, una presión popular que se manifestaba en una conflictiva reivindicación al Ministerio de Educación por parte del movimiento ciudadano de construcciones escolares. En esta situación no tiene nada de extraño que el Ministerio de Educación adelantara, en abril de 1974, a las autoridades de Alcalá, la intención de aprovechar las edificaciones que se habían acondicionado en la antigua Base Aérea, para instalar un nuevo Instituto de Enseñanza Media, y tomara la decisión al respecto en octubre de dicho año.

No obstante, también había contradicciones entre la Dirección General de Universidades y la de Enseñanzas Medias; hasta tal punto que la primera autorizó a la Universidad de Madrid a que ocupara desde octubre de ese año las instalaciones de Alcalá con alumnos de Ciencias Económicas y Empresariales y la segunda decidió que fueran para el Instituto.

Al final, el tema no se resolvió en ningún sentido, porque, dada la disposición de plazas en las facultades y colegios universitarios de Madrid y la incertidumbre sobre lo que Alcalá representaba, abierto el plazo de reservas de plazas para esta última, las peticiones fueron mínimas y, con respecto al Instituto, el Ayuntamiento alcalaíno 
hizo rápidas gestiones para que Enseñanzas Medias dispusiera de terreno en otra parte de la ciudad (en la zona del Val), donde acabó construyéndose el nuevo Instituto Complutense años más adelante

CONTINUARÁ...

En el próximo número: 1975-1977: un proyecto de reapertura al fin con éxito.

\section{NOTAS}

NOTA 19: Ver el Heraldo de Aragón, de 5 de octubre de 1971, también Madrid, 10 de diciembre de 1971.

NOTA 20: Es interesante esta actitud del Ministerio, pero resulta difícil conocer las razones de la misma. Tal vez detrás de toda esa "discreción" está la manifestación de que, como más adelante se plantearía en las luchas políticas dentro de la Universidad, era un grupo de presión - el Opus Dei - el que estaba detrás de todo este proyecto, buscando establecer centros de enseñanza superior con fondos públicos, pero académicamente controlados por los miembros del grupo. Pero tal vez, sin contradecir lo anterior, el director general era víctima de su propia negativa a "conceder" nuevas facultades a otras universidades, como manifestó abiertamente en el Consejo de rectores reunido en Oviedo en septiembre, razón para actuar discretamente en este "favoritismo" a Madrid. De todas formas, quienes conocieron los últimos años del franquismo $\mathrm{y}$, en particular, la Universidad, saben perfectamente que tras cada proyecto estaba la mano de alguno de los "grupos de presión", que buscaban fortalecer sus posiciones en un régimen que no tenía ya ni coherencia en el mantenimiento del poder. 
NOTA 21: Del informe citado de Asela SANZ y Fco. Javier GARCÍA GUTIÉRREZ.

NOTA 22: PRADOS DE LA PLAZA, Luis: ABC; 4 de octubre de 1973. Edición de la mañana, pág. 53.

NOTA 23: Como botones de muestra de esa excepcionalidad estuvieron la decisión de cambiar el calendario académico universitario (comienzo de las clases cada año en el mes de enero) y la convocatoria de un concurso nacional para el nombramiento de los profesores ayudantes de cualquier departamento universitario.

NOTA4 de Vivat Academia: Para aquellos que vivieron estos hechos, hoy desmemoriados, y, para los que han conocido una Universidad de Alcalá ya consolidada, conviene hacer hincapié en estos hechos, ya que significan que el proyecto de recuperación de Alcalá como ciudad universitaria, con sus edificios históricos incluidos, forma parte de una lucha muy antigua y anterior a aquellos que actualmente se colocan medallas que sólo les corresponden en parte. Y lo que es peor, han ignorado sistemáticamente a sus antecesores en la idea, que trabajaron mucho más duro y en peores condiciones para conseguir la realidad que ahora vivimos. 